\title{
Advanced Simulation of Metal Additive Manufacturing Using Maraging Steel and Nickel Alloy
}

Pavel Hanzl, Ivana Zetková, Milan Daňa, Martin Nozar

Faculty of Mechanical Engineering, University of West Bohemia. Univerzitní 2732/8, 30614 Pilsen. Czech Republic. E-mail: hanzlp@rti.zcu.cz, zetkova@rti.zcu.cz, danam@rti.zcu.cz,nozar@rti.zcu.cz

The melting of metal powder by a laser beam is a complex physical metallurgical process and successful processing of the whole Metal Additive Manufacturing (MAM) procedure requires complex management. Therefore, a successful result is not guaranteed. The print job is designed based on the designer's personal experience, and the possibility to verify the correctness of a job proposal would save time and money. This paper deals with demonstrations of print task simulations. Two competing software were used to predict the crash print job. The simulations of both SW solution did not correspond to the real printing result. Possible causes of simulation inaccuracies are listed. The results from actual simulations are useful as support for the print job designer, but they do not completely substitute for real production tests of parts.

Keywords: Simulation, Direct Melting Laser Sintering, Metal Additive Manufacturing

\section{Introduction}

There are plenty of production technologies and each one has its pros and cons. Turning or milling works on the principle of material removal using a cutting tool. The disadvantage is the high material consumption and demands on the multi-axis control of the toolpath and the console of the machine, but the machining result is relatively predictable. In contrast, Metal Additive Manufacturing (MAM) is very rapid in terms of making new parts and does not remove metal material but adds material in layers. MAM powder bed systems are one of three approaches of the material feed stock. There are two other approaches: powder feed systems and wire feed systems. DMLS technology uses the powder bed approach. The metal material in the form of a fine powder is applied (ranked) across the working space and a powerful laser beam melts the powder in areas destined to form the desired shape. [3]

However, this method generates high residual stress in the molten material. The parts must be oriented and adequately attached to the building platform by support structures to suppress the stress. These structures are a problem because they have to be removed after production and the quality of the attached surfaces deteriorates. They also waste expensive metal powder. Therefore, there is a general effort to reduce these supports to a minimum. [4][5]

This is the job of the print job designer, who seeks a balance between manufacturability, financial demands of production, part deformation and heat transfer. The design process is mostly based on previous experience, although this is the most influential part of the manufacturing process. [1] However, new precise methods have become available with the development of specialized software. [2]

Several articles dealt with different methods of simulation MAM. Lindgren et al. [6] describes the application of modelling approaches used in Computational Welding Mechanics (CWM) applicable for simulation MAM. They focused on the approximation of the behaviour in the process zone and the solid material, particularly in the context of changing microstructure. The CWM application to direct energy deposition shows that these techniques apply very well to MAM processes. However, there is a need to resort to simplification.

Hodge et al. [7] consider of the complex interaction between heat transfer and solid mechanics. Both incremental deformation and stresses generated by various simply model runs were compared to experimental results. The comparisons were generally encouraging.

This paper looks at the credibility of various solutions which work on different principles. The first simulation tool works on incremental strain in layers. This software is universal for all metal powder and it should be useable in a short time. It is necessary only to provide a calibration based on a test print. This step reflects the setup of the printing machine and the metal powder used. This method is time-saving, unlike other kinds of software which work with exact values. These values must first be obtained by experimental measuring. Some suppliers are considering this, and their solutions have profiles for many devices and materials. The second method works with these profiles with pre-defined constants. This solution does not exactly reflect the characteristics of the specific production machine because the characteristics of each AM 
machine deviate from typical averages for a specific model.

Experiments proved that the slightest deviations of the settings from the real values of the material and machine constants can mean differences in credibility of the simulation outputs. The results listed in this paper are not fully comparable. They are rather informative because the simulations depend on the proper setup of their constants. In addition, each kind of software takes a different approach to the issue.

\section{Printing parameters}

The comparison parts for testing accuracy of simulation were produced on an EOS M 290. This device uses an $\mathrm{Yb}$-fibre laser for melting metal powder with a maximum power of $400 \mathrm{~W}$. The processing conditions were predefined by EOS. A solid ceramic recoater blade was used to apply thin $40 \mu \mathrm{m}$ layers.

The same printing parameters for melting of EOS MaragingSteel and EOS NickelAlloy IN718 were following: scan speed $960 \mathrm{~mm} / \mathrm{s}$, laser power $285 \mathrm{~W}$, distance between tracks $0,11 \mathrm{~mm}$, stripe width $10 \mathrm{~mm}$, stripe overlap $0,08 \mathrm{~mm}$. Each layer was rotated $33^{\circ}$ relative to the previous one.

The material composition of EOS MaragingSteel (W.Nr 1.2709) and EOS NickelAlloy (W.Nr 2.4668) are shown in Table 1 and 2.

Tab. 1 The material composition of EOS MaragingSteel MS1, European standard 1.2709 [7]

\begin{tabular}{ccccccccccccc}
\hline Wt [\%] & $\mathrm{Ni}$ & $\mathrm{Co}$ & $\mathrm{Fe}$ & $\mathrm{Mo}$ & $\mathrm{Ti}$ & $\mathrm{Al}$ & $\mathrm{Cr}$ & $\mathrm{Mn}$ & $\mathrm{P}$ & $\mathrm{S}$ & $\mathrm{C}$ & $\mathrm{Si}$ \\
\hline $\mathrm{MS1}$ & $17-19$ & $8.5-9.5$ & rest & $4.5-5.2$ & $0.6-0.8$ & $0.05-0.15$ & $<0.5$ & $<0.1$ & $<0.01$ & $<0.01$ & $<0.03$ & $<0.1$ \\
\hline
\end{tabular}

Tab. 2The material composition of EOS NickelAlloy, European standard $2.4668 \quad[8]$

\begin{tabular}{lcccccccccccc}
\hline Wt [\%] & $\mathrm{Ni}$ & $\mathrm{Cr}$ & $\mathrm{Fe}$ & $\mathrm{Nb}$ & $\mathrm{Mo}$ & $\mathrm{Ti}$ & $\mathrm{Al}$ & $\mathrm{Co}$ & $\mathrm{Cu}$ & $\mathrm{Si}$ & $\mathrm{Mn}$ & $\mathrm{Ta}, \mathrm{C}, \mathrm{S}, \mathrm{P}$ \\
\hline $\mathrm{IN} 718$ & $50-55$ & $17-21$ & rest & $4.8-5.5$ & $2.8-3.3$ & $0.65-1.15$ & $0.2-0.8$ & 1.0 & 0.30 & 0.35 & 0,35 & $<0.17$ \\
\hline
\end{tabular}

\section{Method and Calibration}

In the first type of simulation, a calibration print is necessary to provide a proper result. The calibration part is positioned in the directions of the machine axes $\mathrm{x}, \mathrm{y}$ and $45^{\circ}$. After printing, the calibration part is cut longitudinally up to the clamping part to the building

\section{EOS NickelAlloy IN718}

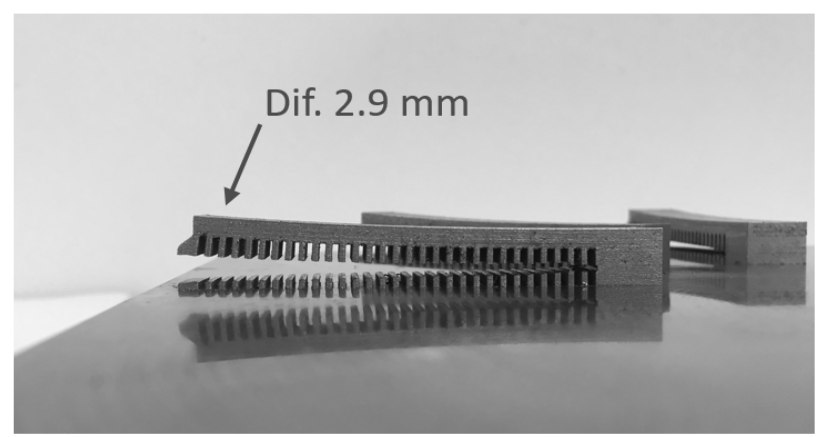

platform. Then, the deflection of the upper edge relative to the original state is measured. The height differences reflect the states of residual stress that arose in $\mathrm{x}, \mathrm{y}$ and $45^{\circ}$ directions. This deflection is influenced by a number of factors such as process parameters, characteristics of the MAM device, the material and its particle distribution, including particle shape. The measured differences are shown in Figure 1.

\section{EOS MaragingSteel MS1}

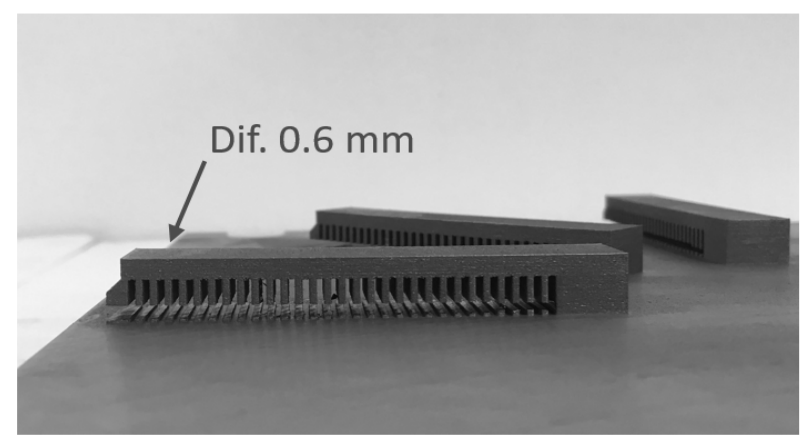

Fig. 1 The deflection after cutting of the calibration part for EOS NickelAlloy IN718 and EOS MaragingSteel MS1

The material IN718 showed a higher value of deviation than MS1 which points to higher residual stress in the printing material. The deviations for MS1 were $0.51 \mathrm{~mm}, 0.62 \mathrm{~mm}$ and $0.56 \mathrm{~mm}$ in directions $\mathrm{x}$, $45^{\circ}$ and y respectively. The measured deviations are used for specifying the strain $\varepsilon$ in selected directions. The simulation works only for these values. No other parameters are considered in this version such as temperatures, utilization of the platform area by parts, etc.

In the second type of simulation, the solver performs a thermo-mechanical analyses. It works with the relevant process parameters. The benefit of this solution is that heat transfer is considered. However, each MAM device has slightly different characteristics that are not initially considered. This can be overcome by gradually refining the input constants. This is however time-consuming.

\section{Simulations}

\section{Turbine blade}

The first example part is a gas turbine blade with dimensions $77 \mathrm{~mm}$ × $36 \mathrm{~mm}$ × $27 \mathrm{~mm}$. The building 
direction is along the longest dimension. Part orientation with the designed support structures is shown in Figure 2. The blade was produced by an EOS M290 printer. The material was maraging steel 1.2709 according to European classification. Two identical

Turbine Blade Nr.1

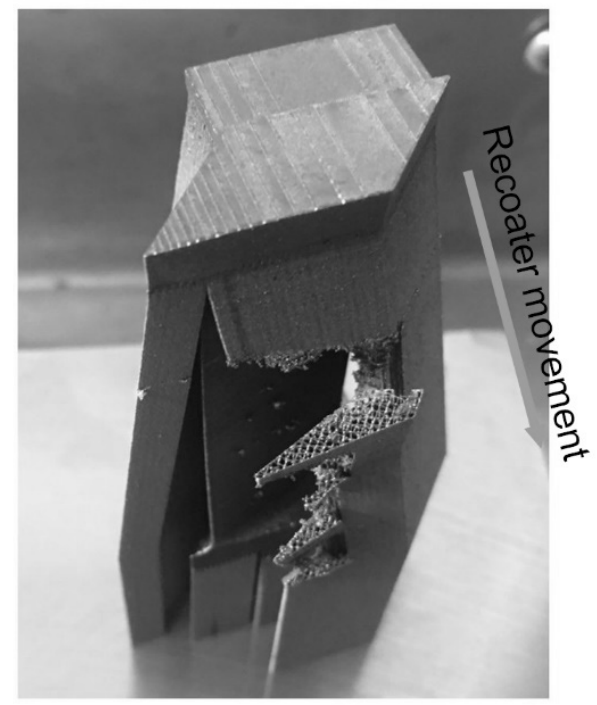

models were printed simultaneously. Both parts showed similar defects. The support structure is a good input to verify the accuracy of simulations.

\section{Turbine Blade Nr.2}

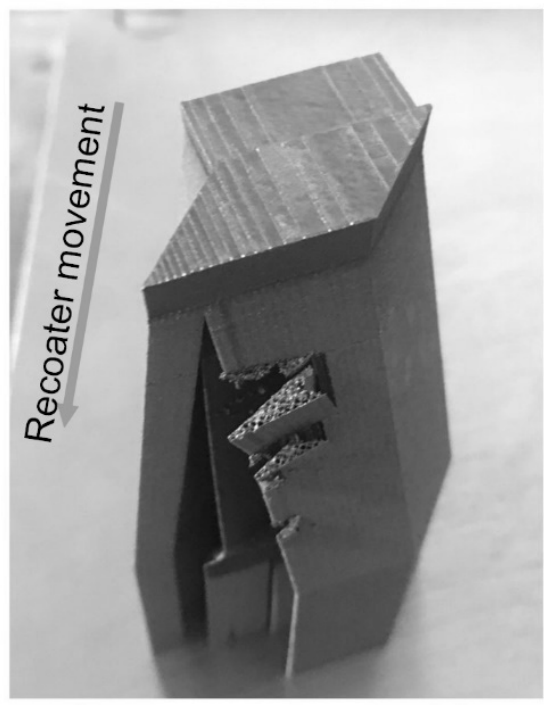

Fig. 2 Printed turbine blades from MS1 steel with the same repeated defect

The expression of the model in the analysis software is by voxels. The voxel size is set at $0.8 \mathrm{~mm}$ for the first solver. The part's orientation on the platform has been taken into account. The selected results are shown in Figure 3. Other results are available such as displacement, stress, etc. However, the analyses do not

Model with Supports
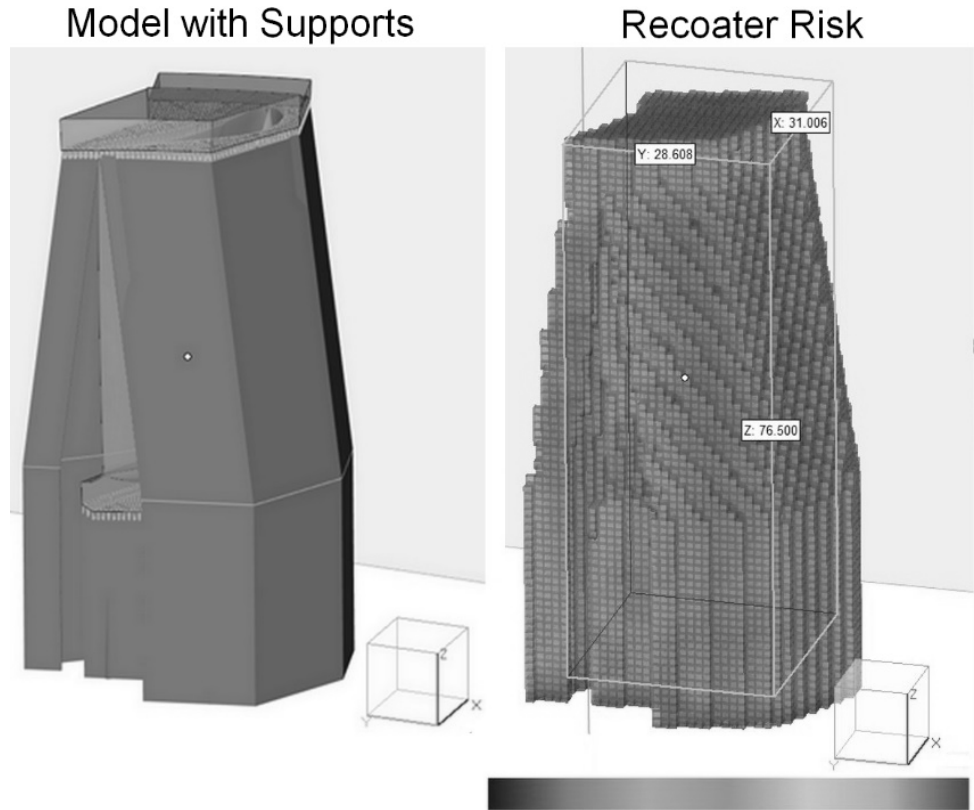

$0 \mathrm{~mm} 0[-]$

$-0.02675 \mathrm{~mm}$ indicate any possible risks associated with production by MAM. The only visible risk area is in the anchoring portion of the support structure to the building platform and in a small segment of the blade edge, but there were no real production problems. The real risk areas were not detected (see Figure 2).

Fig. 3 Analysis of turbine production from MS1

Failure Criteria

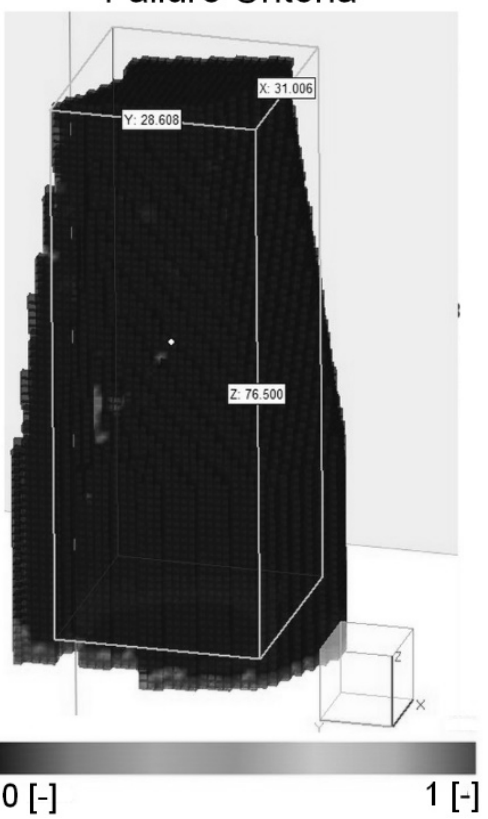

\section{Holder}

The second part, a holder, for verification, was made of Inconel 718. This holder has thin walls. The voxel size was set at $0.7 \mathrm{~mm}$. The simulations were provided by the first solver and the results are shown in Figure 4. These correspond more with real production, in which defects appeared as seen in Figure 5. 

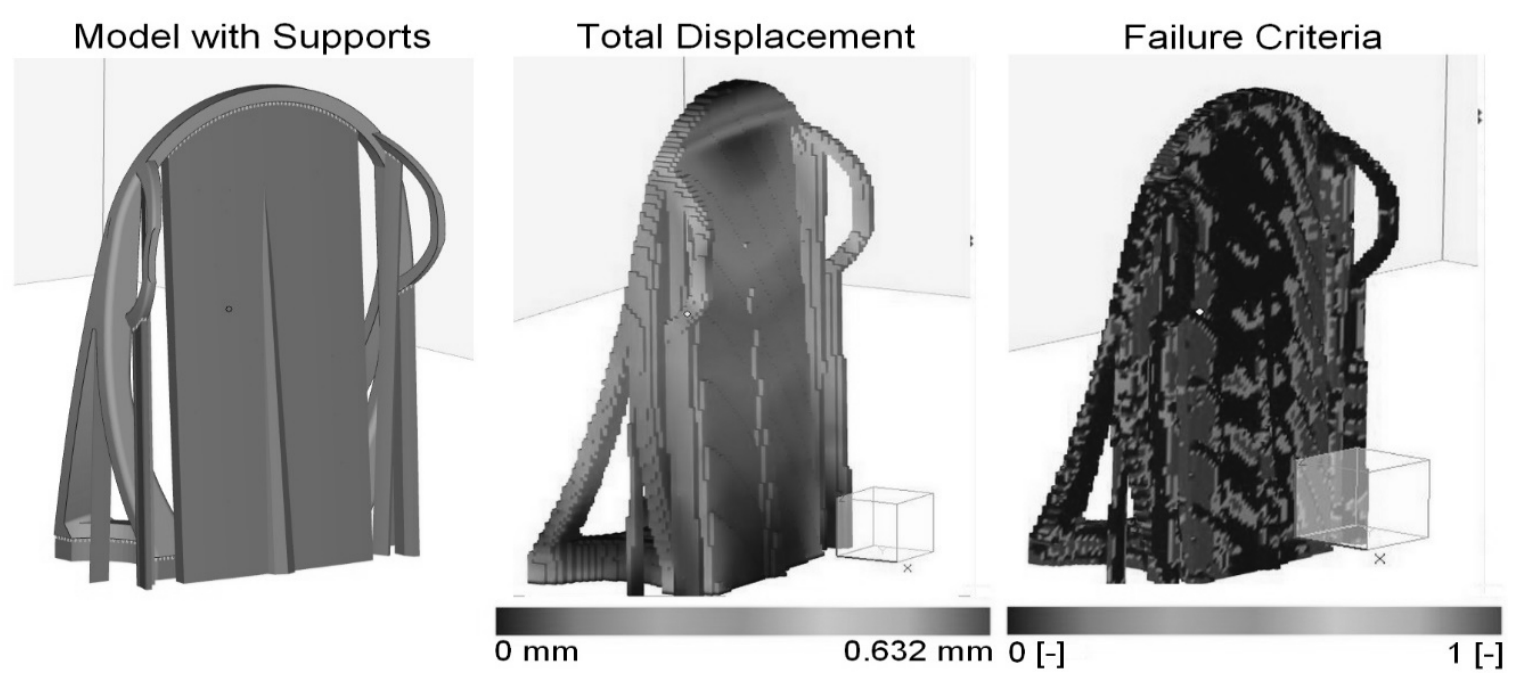

Fig. 4 The results of holder analysis, IN718 material

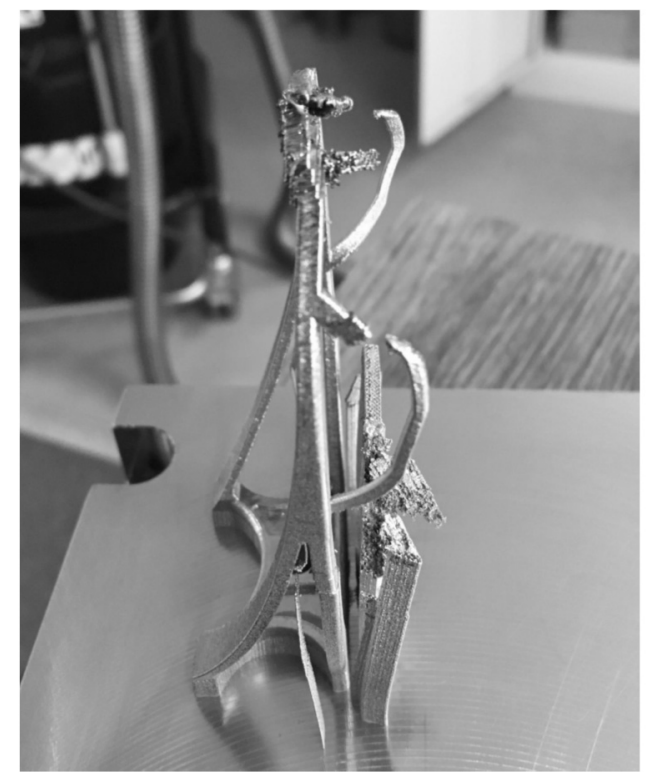

Fig. 5 The real state of the printed holder

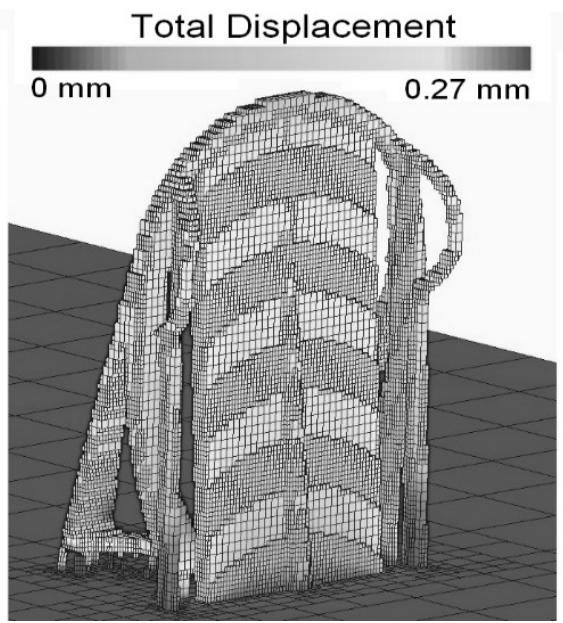

This damaged holder was analysed by the second simulation solver. The voxel size was adaptive with $0.05 \mathrm{~mm}$ padding tolerance. Outputs such as stress, strain, deformation and temperature history are available. This, unlike the previous one, works with explicit values of process parameters of MAM. A default profile for IN625 (250 W, $800 \mathrm{~mm} / \mathrm{s}$ and $40 \mu \mathrm{m})$ was used, because the material setting for IN718 was not allowed due to limited access to the SW licence. This profile contains many parameters. Some may be correlated with temperature, for example, strength. This allows high theoretical precision of simulation. On the other hand, refining the simulation would require extensive measurement of sensitive properties of the material used for MAM. The second solution was disadvantaged by the limited material selection for this experiment. However, the simulation of the holder production was provided with similar material and showed no potential risks (see Figure 6).

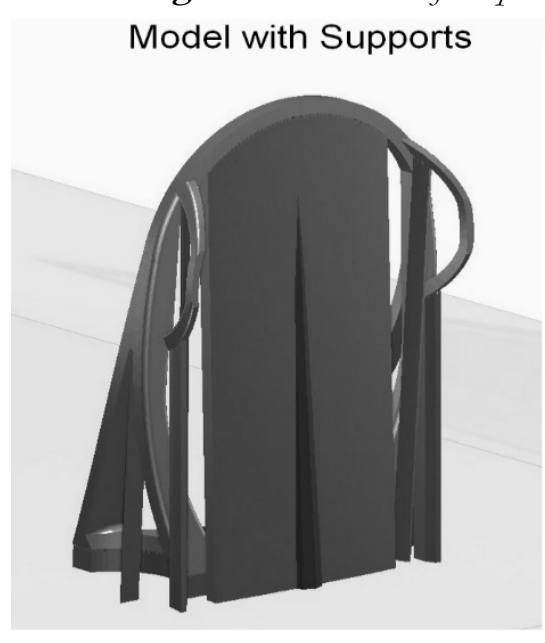

Fig. 6 The outputs of the second testing solver

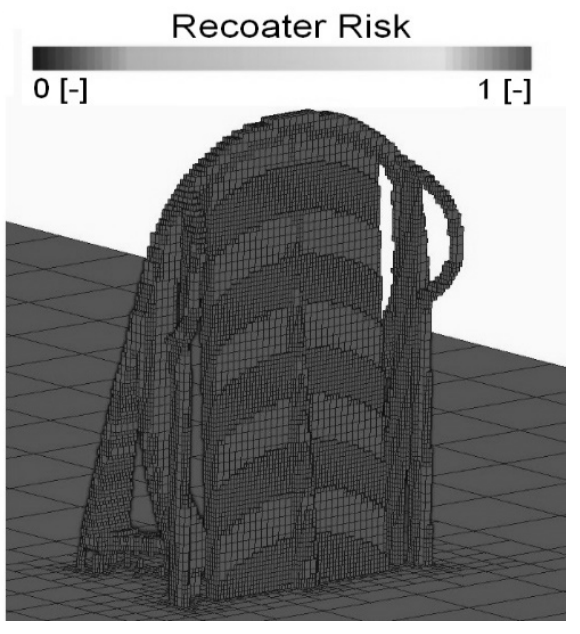

\section{Conclusion}

This paper deals with predicting the success of printing jobs with two different solvers. The results of the analyses were verified on real printed parts. Both solutions can be used to guide decisions about part orientation, support structure design and placement. In the second solver, the temperature history can be 
used to investigate the potential quality of parts made with the setting of the processing parameters and the use of a particular material.

Experiments confirmed that the metal additive manufacturing process using a laser beam for melting is hard to predict. In particular, the behaviour of the maraging steel did not correspond with the real behaviour of MAM. The prediction of the part made from IN718 was closer to reality. This is due to the instability of residual stress in laser melted maraging steel. The stress level in maraging steel is lower, as is demonstrated by the smaller deflection of the calibration part in Figure 1.

The calibration jobs reflect the quantity of the residual stress. More minor deviations during the calibration of the SW solver were measured for the MS1 material than for IN718. The higher quantity of the residual stress which is trapped in the Inconel alloy, the more favourable it is to the simulations. Therefore, the behaviour of the IN718 material is more predictable and the simulation achieves better accuracy than for MS1 material.

The thermal load of a printed part depends on the print height and the melted area content of the layer. This leads to a different residual stress in the same part when a different number of parts are printed during one print job. Temperatures in the first solver were not considered completely. Therefore, the variability of residual stress due to local overheating was not considered during the simulation. The version used does not support a temperature analysis which could be added in a future version. The simulations of the first solver work only based on the incremental displacement of individual layers. The second solver can provide information about temperatures during the printing process and the overheated areas can be detected.

Usually the simulation of the temperature history needs more time to compute. It is important to keep in mind that metal additive manufacturing using a laser beam for melting is a complex process and the simulations work with simplified physical models. As the number of variables increases, the analysis will be longer, and the calculation requirements disproportionately increase.

The result of the analyses is that actual simulations are useful as software tools for the print job designer. But they do not substitute for real manufacturing tests of parts and personal experience using this manufacturing technology. These software tools still undergoing development, resulting in increasing accuracy. In this context, it should be mentioned that this paper reviewed software versions available in 2019.

\section{Acknowledgement}

The support of the grant TH02010303 of the

\section{Czech Technology Agency is acknowledged.}

\section{References}

[1] HRDLICKA, F., KRATOCHVIL, M., MAZINOVA, I., FLORIAN, P. (2019) Use of Material-Shape Factors in Mechanical Design, In: Manufacturing Technology, ISSN 1213-2489, June 2019, Vol. 19, No. 3, Pp. 397-403

[2] KING, W., ANDERSON, A.T., FERENCZ, R.M., HODGE, N.E., KAMATH, C., KHAIRALLAH, S.A. (2016) Overview of modelling and simulation of metal powder bed fusion process at Lawrence Livermore National Laboratory, Materials Science and Technology, Vol. 31, No.8, Pp. 957-968, DOI: 10.1179/1743284714Y.000000072

[3] FOUSOVÁ, M., VOJTĚCH, D., FOJT, J. (2016) Microscopic evaluation of 3D-printed materials surface and characteristic microstructure, In: Manufacturing Technology, ISSN 1213-2489, October 2016, Vol. 16, No. 5, Pp. 902-909

[4] MORGAN, D., AGBA, E., HILL, CH. (2017) Support Structure Development and Initial Results for Metal Powder Bed Fusion Additive Manufacturing, Procedia Manufacturing, ISSN 2351-9789, 2017, Vol. 10, Pp. 819-830

[5] DAŇA, M., ZETKOVÁ, I., HANZL, P. (2019) The Influence of a Ceramic Recoater Blade on 3D Printing using Direct Metal Laser Sintering, In: Manufacturing Technology, ISSN 1213-2489, February 2019, Vol. 19, No. 1, Pp. 23-28

[6] LINDGREN, L.E., LUNDBÄCK, A., FISK, M., PEDERSON, R., ANDERSSON, J. (2016) Simulation of additive manufacturing using coupled constitutive and microstructure models, Additive Manufacturing, Volume 12, Part B, 2016, Pages 144-158, ISSN 2214-8604

[7] HODGE, N.E., FERENCZ, R. M., VIGNES, R.M. (2016) Experimental comparison of residual stresses for a thermomechanical model for the simulation of selective laser melting, Additive Manufacturing, Volume 12, Part B, 2016, Pages 159-168, ISSN 2214-8604

[8] EOS MaragingSteel MS1, eos.info[online]. [date $24^{\text {th }}$ june 2020] available from: https://www.eos.info/en/additive-manufacturing/3d-printing-metal/dmls-metal-materials/tool-steel

[9] EOS NickelAlloy IN718, eos.info[online]. [date $24^{\text {th }}$ june 2020] available from: https://www.eos.info/en/additive-manufacturing/3d-printing-metal/dmls-metal-materials/nickel-alloys 